Article

\title{
Active Continuous-Flow Micromixer Using an External Braille Pin Actuator Array
}

\author{
Yawar Abbas ${ }^{1,4} *$, Junichi Miwa ${ }^{1}$, Roland Zengerle ${ }^{1,2,3}$ and Felix von Stetten ${ }^{1,2}$ \\ 1 Laboratory for MEMS Applications, Department of Microsystems Engineering, IMTEK, University \\ of Freiburg, Georges-Koehler-Allee 103, 79110 Freiburg, Germany; \\ E-Mails: miwajunichi@gmail.com (J.M.); zengerle@imtek.uni-freiburg.de (R.Z.); \\ vstetten@imtek.uni-freiburg.de (F.V.S) \\ 2 \\ HSG-IMIT, Institut für Mikro- und Informationstechnik, Georges-Koehler-Allee 103, 79110 \\ Freiburg, Germany \\ 3 BIOSS - Centre for Biological Signalling Studies, University Freiburg, 79104 Freiburg, Germany \\ 4 BIOS-Lab on chip group, MESA+ Institution of Nanotechnology, University of Twente, $7522 \mathrm{NH}$ \\ Enschede, The Netherlands
}

* Author to whom correspondence should be addressed; E-Mail: y.abbas@ utwente.nl;

Tel.: +31-53-489-2604; Fax: +31-53-489-3595.

Received: 17 December 2012; in revised form: 11 February 2013 / Accepted: 1 March 2013 /

Published: 14 March 2013

\begin{abstract}
We present a continuous-flow active micromixer based on channel-wall deflection in a polydimethylsiloxane (PDMS) chip for volume flows in the range up to $2 \mu \mathrm{L} \mathrm{s}^{-1}$ which is intended as a novel unit operation for the microfluidic Braille pin actuated platform. The chip design comprises a main microchannel connected to a series of side channels with dead ends aligned on the Braille pins. Computer-controlled deflection of the side-channel walls induces chaotic advection in the main-channel, which substantially accelerates mixing in low-Reynolds number flow. Sufficient mixing (mixing index MI below 0.1 ) of volume flows up to $0.5 \mu \mathrm{L} \mathrm{s}^{-1}$ could be achieved within residence times $\sim 500 \mathrm{~ms}$ in the micromixer. As an application, continuous dilution of a yeast cell sample by a ratio down to 1:10 was successfully demonstrated. The mixer is intended to serve as a component of bio-analytical devices or as a unit operation in the microfluidic Braille pin actuated platform.
\end{abstract}

Keywords: active micromixer; continuous-flow; chaotic advection; channel wall deflection; PDMS chip; cell sample dilution; microfluidic Braille pin actuated platform 


\section{Introduction}

Mixing of fluids in microchannels is challenging due to the stable laminar flow. The speed of mixing is limited by molecular diffusion, and is inversely proportional to the size of the fluid molecule or particle to be mixed [1]. Typically, for relatively large biomolecules or even cells, mixing can amount to hours in microchannels. An efficient method for mixing enhancement in microchannels is needed for bio-analytical applications such as cell counting, enzyme assays, screening assays, cell lysis, protein folding, and many others [2].

In recent years, several active and passive mixers have been designed, based on the idea of reducing characteristic diffusion length in microchannels [3]. One profound phenomena for efficient microscale mixing is chaotic advection [4], which involves local stretching and folding of the fluid streams and results in significant reduction in effective diffusion length. The key to effective mixing lies in the generation of strong stretching and folding [5]. Chaotic advection is induced in passive mixers through channel geometries [6-9], whereas in active mixers, stretching and folding is introduced with the aid of external perturbation sources [10-12]. Active micromixers generally provide enhanced control over the process for a broader range of flow conditions [11,12]. Several active mixers based on chaotic advection have been reported where efficient mixing was observed in sub-seconds [4,5,11]. Most of the previously reported active mixers have been fixed-volume mixers. Mixing in continuous-flow condition is challenging due to the lower speed of mixing compared to the typically short residence time of the particles in the mixer. Tabeling et al. [4] reported a cross-channel micromixer which exploits chaotic motion of fluid particles in the main channel due to pressure perturbation at side channels. Such structures with robust and compact pressure-perturbation sources can be used for rapid mixing in microscale for continuous flow condition.

In this work we present a continuous-flow active micromixer that creates chaotic advection in crosschannel structures with the aid of mechanical actuation of polydimethylsiloxane (PDMS) membranes. The mixer is a novel unit operation for the Braille pin controlled microfluidic platform first described by $\mathrm{Gu}$ et al. [13] and Erickson [14].

\section{Material and Method}

\subsection{Principle of Braille Pin Controlled Continuous Micromixer}

The novel continuous-flow active micromixer creates chaotic advection in cross-channel structures with the aid of mechanical actuation of PDMS membranes. The main microchannel is connected to a series of side channels (cross-channel structures) with dead ends that are aligned on Braille display pins. The side channel walls deform as the Braille pins underneath deflect, creating transverse flow at the mixing fluid interface in the main channel. By operating the Braille pins in a periodic sequence, the fluid undergoes local stretching and folding, which leads to rapid fluid mixing. Figure 1 depicts the principle of chaotic advection in the main channel due to pressure perturbation in the side channels. 
Figure 1. Schematic of a cross-channel mixer and principle of pressure perturbation. (a) At no actuation, flow in main channel is laminar. (b) After Braille pin actuation stretching and folding of fluid interface occurs in main channel.

(a)

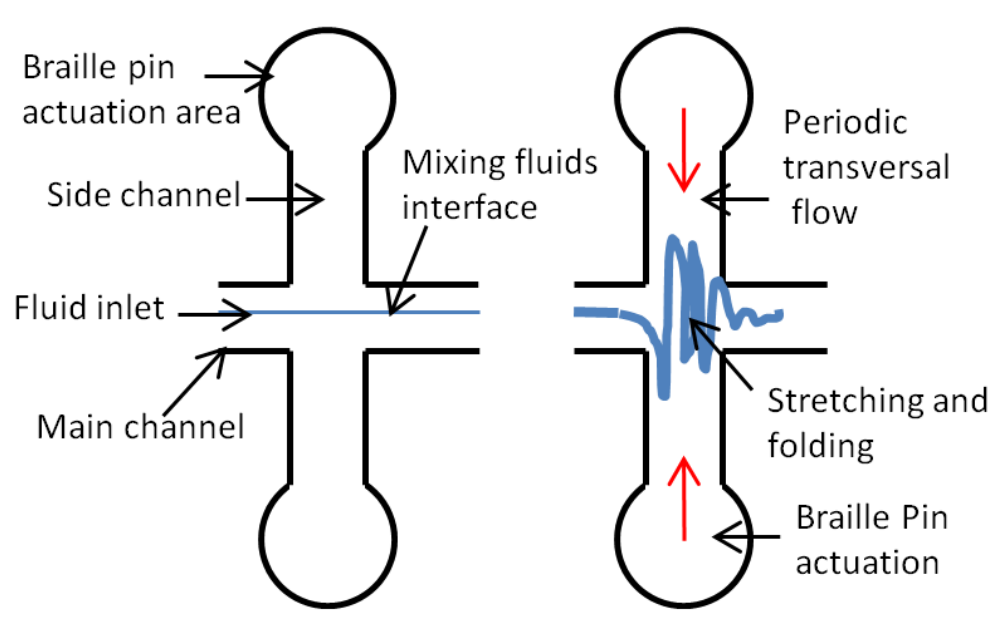

\subsection{Chip Fabrication}

The microfluidic chip is composed of polydimethylsiloxane (PDMS) fabricated using standard soft lithography procedures [15]. Figure $2 \mathrm{a}$ shows the schematic of the chip fabrication process. The master mould structure was fabricated on a 4-inch silicon wafer patterning multi-layered photoresist (AZ9260, Microchemicals $\mathrm{GmbH}$ ). The patterned $100-\mu \mathrm{m}$ thick structures were reflowed at $120{ }^{\circ} \mathrm{C}$ for $2 \mathrm{~h}$ to obtain round channel cross sections. The PDMS channel structures were made by pouring Sylgard 184 (Dow Corning Corp., mixture ratio of curing agent to pre-polymer 1:10) onto the mould and baking in an oven at $70{ }^{\circ} \mathrm{C}$ for $2 \mathrm{~h}$. A $100-\mu \mathrm{m}$ thick PDMS membrane was spun on another silicon wafer and cured in the same oven to form the deformable membrane. The two PDMS layers were permanently bonded after surface activation in oxygen plasma. Figure $2 b$ shows the PDMS chip after fabrication. The microfluidic channel depth and width are $100 \mu \mathrm{m}$ and $200 \mu \mathrm{m}$, respectively, and the dead-end chamber (round area) for Braille-pin actuation has a diameter of $1.5 \mathrm{~mm}$. The resulting volume of the mixers fluid channel is $0.15 \mu \mathrm{L}$, the dead volume of one side arm is $0.19 \mu \mathrm{L}$ and the displaced volume upon one braille pin is $0.16 \mu \mathrm{L}$. 
Figure 2. (a) Polydimethylsiloxane (PDMS) chip fabrication steps. (b) Snapshot of the microfluidics chip after fabrication, magnified view of one cross-channel structure in the PDMS chip shows the cross-channel with round area at the dead end. Braille pins deflect the membrane at the round areas.

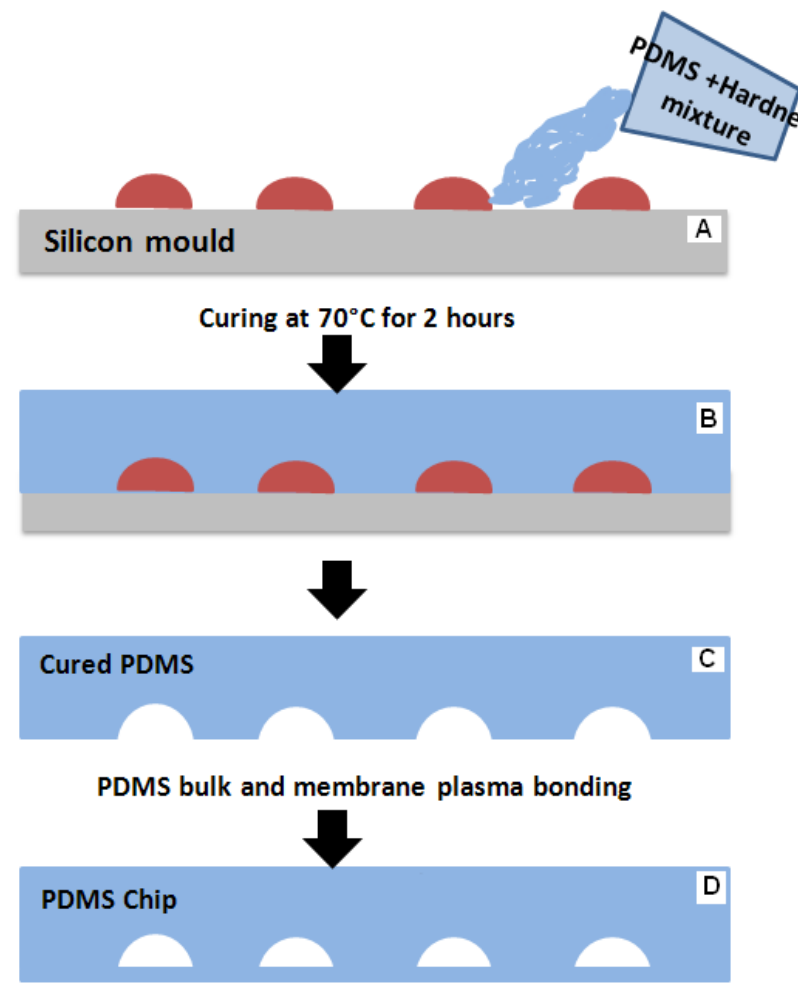

(a)

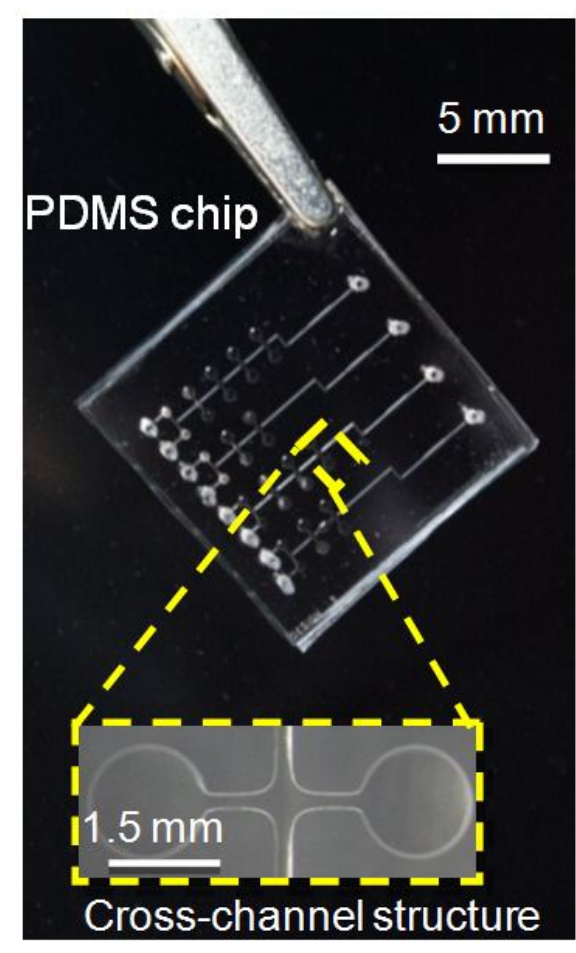

(b)

\subsection{Braille Pin Actuator}

The feasibility of Braille pin device as a mechanical actuator for fluid metering in bio-analytical applications has been demonstrated by several groups $[13,16]$. We used a commercial Braille display (KGS Corp., Saitama, Japan) that provides an $8 \times 8$ array of $1.3 \mathrm{~mm}$ diameter pins that are each connected to piezoelectric bimorphs. These pins each deliver a force of $0.1 \mathrm{~N}$ for membrane deflection of up to $700 \mu \mathrm{m}$. The force corresponds to approximately $280 \mathrm{kPa}$ pressure at the PDMS chambers when the chip is aligned and clamped on the Braille display (Figure 3b). The resonance frequency of each piezoelectric element is $10 \mathrm{~Hz}$. The pin actuation is controlled by a computer interface connected through an electronic interface circuit manufactured by Biofluidix GmbH, Freiburg, Germany [17].

\subsection{Experimental Setup}

A schematic of the experimental setup is illustrated in Figure 3a. The membrane side of the PDMS chip is placed directly on top of the Braille display pin after careful alignment of the dead-end chambers with the Braille display pins. To pump the fluid through the chip at a precisely predetermined flow rate, a commercial syringe pump (neMESYS, Cetoni GmbH, Korbussen, Germany) was used. A high-speed camera (pco. 1200, PCO AG, Kelheim, Germany) was used to acquire 
instantaneous images of the fluid mixing inside the fluid channels. A $12 \times$ objective lens (Navitar Inc., New York, NY, USA) was attached to the high-speed camera to magnify the images.

Figure 3. (a) Schematic of complete experimental setup. (b) PDMS chip with integrated cross channel and inlet/outlet tubes on a commercially available Braille pin actuator.

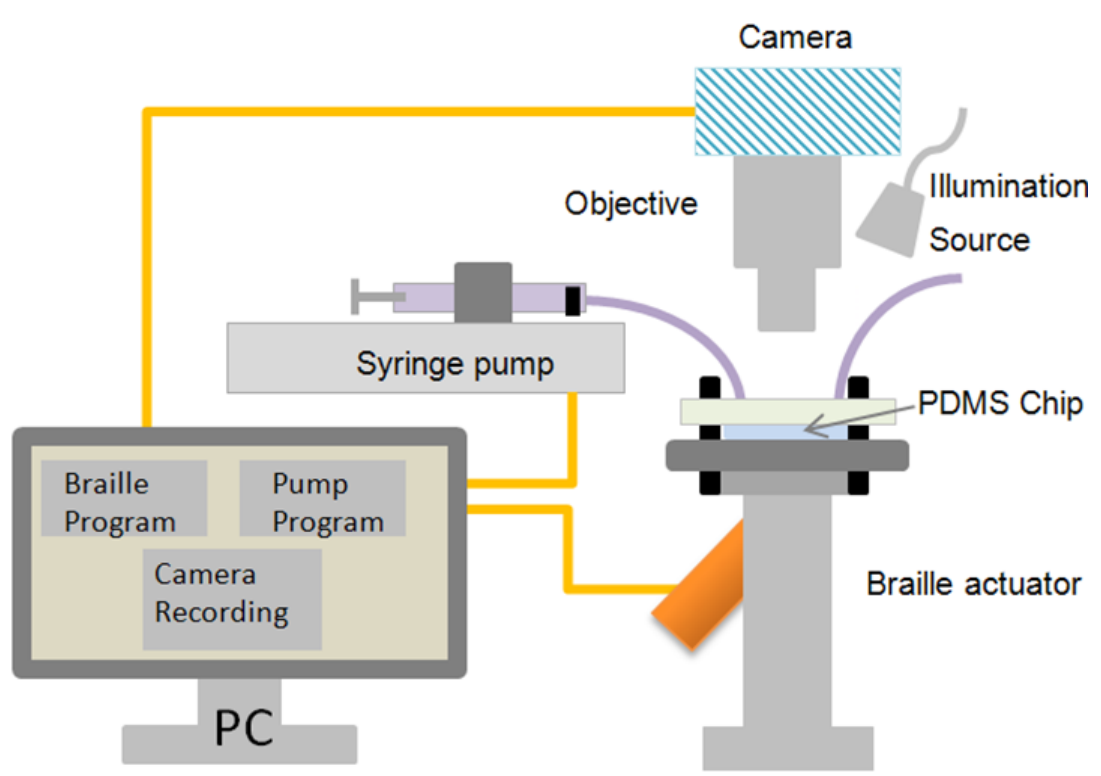

(a)

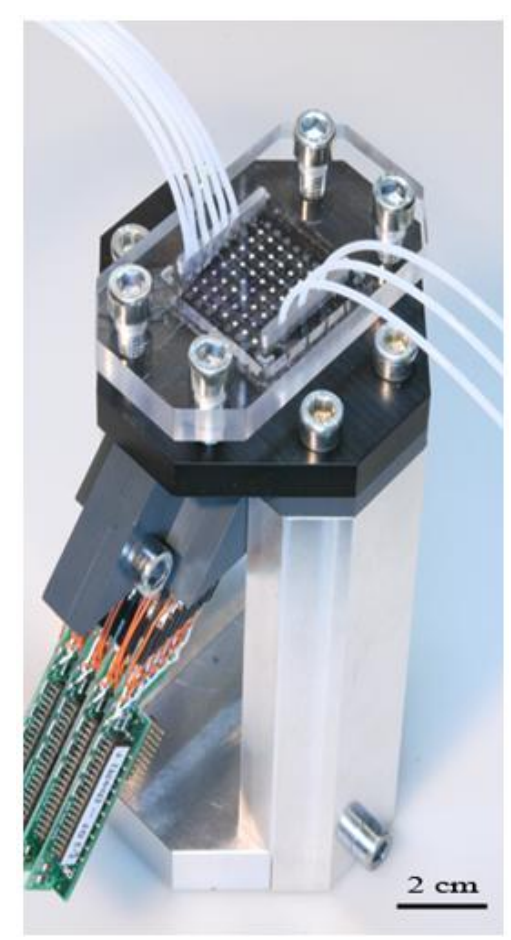

(b)

\subsection{Mixing Quantification Technique}

The efficiency of the Braille-actuated micromixer is evaluated by image analysis of instantaneous images of two water streams, one of which is colored with ink. The performance is quantified using a mixing index (MI) defined as the standard deviation of the pixel intensity values of an instantaneous image at time $t$ from a certain reference image. In this study, the reference is defined as the image where the fluid is homogenized or completely mixed [11]. The expression for MI is given in Equation (1).

$$
M I=\sqrt{\frac{1}{N} \sum_{n=1}^{N}\left(\frac{I_{(t, n)}-I_{\text {avg }}}{I_{\text {avg }}}\right)^{2}}
$$

where $N$ is number of pixels and $I$ is the optical intensity. MI values of 1 and 0 indicate perfect laminar distribution and completely mixed state, respectively. Many of the literature consider $0.1 \mathrm{MI}$ as a threshold value to define well mixed states $[11,18]$. Therefore, mixing time, which is the time it takes for the two fluids to be well mixed in the main channel, is defined as the time when the MI reaches 0.1. In case of this continuous micromixer the mixing time is equivalent to the residence time of the fluid in the micromixer which is related to the volume flow and the active volume of the micromixers fluid channel. 


\section{Results and Discussion}

\subsection{Qualitative Analysis}

Figure 4 shows the behaviour of the fluid interface at the cross channel, where the stretching and folding of the fluid interface is observed during pin actuation. Here only one cross-channel structure is shown. The pair of braille pins actuates in alternative sequence i.e., upper pin actuates after the actuation of lower pin or vice versa. With no actuation the ink/DI water interface follows laminar flow, Figure 4a. The dark area in the upper side-channel of Figure $4 \mathrm{c}$ is caused by the flow of ink during the actuation of lower pin in Figure 4b. Stretching and folding is a key indicator of chaotic advection, and this image sequence clearly shows the capability of the Braille-actuated micromixer to perform rapid fluid mixing. As observed by visual inspection, the deflection of membrane is synchronized with the pin actuation for actuation frequencies up to $10 \mathrm{~Hz}$, therefore no superimposed effect of membrane occurs on the mixing efficiency.

Figure 4. Snapshots of the cross-channel structure (top view) during Braille actuation at an interval of $200 \mathrm{~ms}$. (a) Before actuation. (b) Lower Braille pin is actuated and induces transversal flow causing stretching of the fluid interface. (c), (d) Upper pin is actuated, causing the folding of the stretched interface. (Total flow rate in channel is $0.4 \mu \mathrm{L} \mathrm{s}^{-1}$ ).

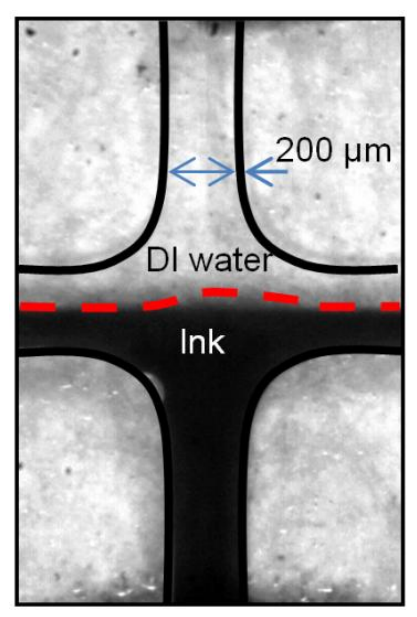

(a)

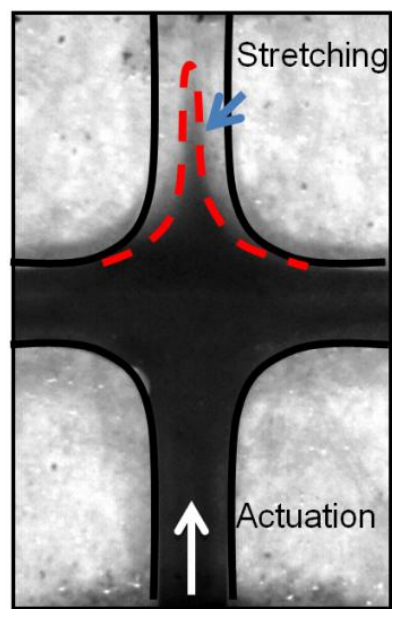

(b)

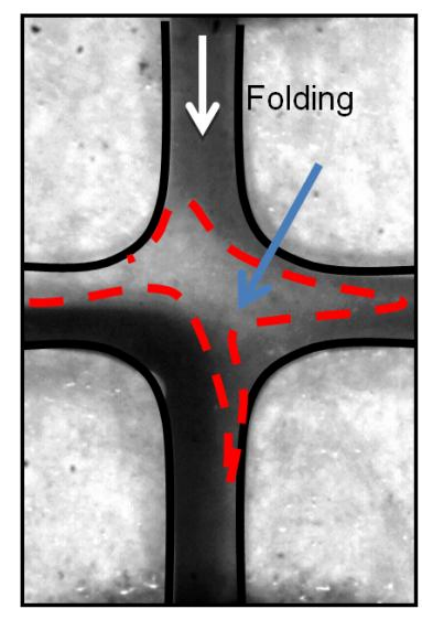

(c)

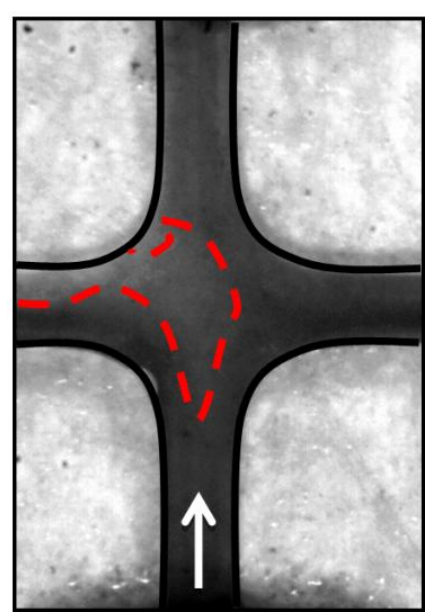

(d)

\subsection{Quantitative Analysis}

For quantitative analysis two cross-channel structures are used during mixing operation. Figure 5a illustrates the effect of pin-actuation frequency on the mixing efficiency. At lower frequencies $(<1 \mathrm{~Hz})$ mixing is not efficient due to weak perturbation; in this case the actuation time is longer than the residence time of the fluid element in the mixing structure. With each pin actuation, $0.16 \mu \mathrm{L}$ of fluid is displaced in the side channel. At $10 \mathrm{~Hz}$ the actuated flow rate in the side channel is $c a .1 .6 \mu \mathrm{L}$. At frequencies exceeding the resonance frequency of the Braille actuator $(>10 \mathrm{~Hz})$, the mixing efficiency decreases due to lower velocities of the displaced fluids in side channels (lower propulsion of pin into the membrane). Therefore the optimal mixing performance is observed at the resonance frequency $(\sim 10 \mathrm{~Hz})$ with the current setup. 
Figure $5 b$ depicts the dependency of mixing efficiency on the total flow rate of fluids inside the main channel. The mixing efficiency decreases with increasing flow rates due to the fact that the residence time of the fluid in the channel is shortened and fluid elements undergo less perturbation before they leave the mixing chamber. Although the Reynolds number of the main-channel flow at $2 \mu \mathrm{L} \mathrm{s}^{-1}$ is relatively high ( 20), it is not high enough to enhance mixing by turbulence. Thus for flow rates higher than $0.4 \mu \mathrm{L} \mathrm{s}^{-1}$, more than two cross-channels structures are required (i.e., Number of actuating pins $>4$ ) for efficient mixing. The ratio of the side-channel flow rate to the main-channel flow rate is also an important parameter to quantify the operational parameter of this mixer. Figure $5 \mathrm{~d}$ depicts the relation between the side-channel flow rates and the main-channel flow rates for sufficient mixing efficiency $(\mathrm{MI}<0.1)$. To achieve sufficient mixing the required side-channel flow rate is almost the square of the main-channel flow rate, as apparent from Figure 5d.

Figure 5. Different effects on the MI of the cross-channel mixer. (a) Effect of actuation frequency of braille pin actuator. (b) Effect of total flow velocity of fluids in main channel. (c) Effect of fluid viscosity. (d) Relation between the main-channel flow rates and the side-channel flow rates for acquiring mixing index within 0.1 .

(a)

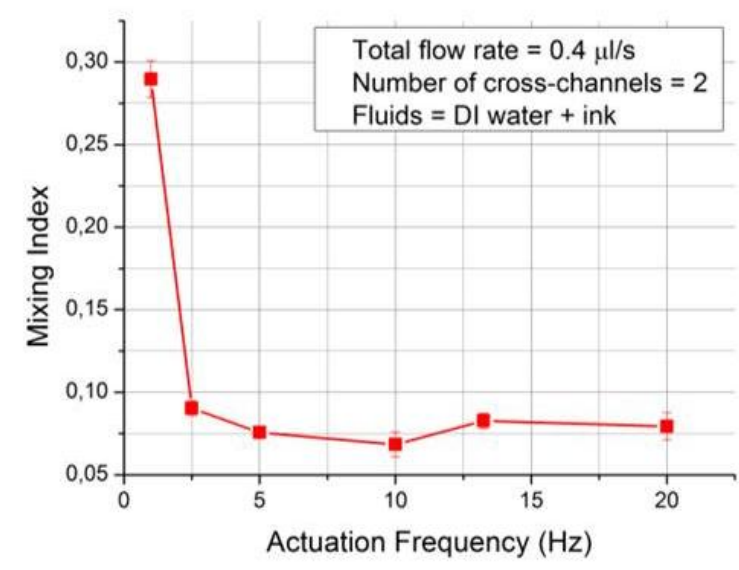

(c)

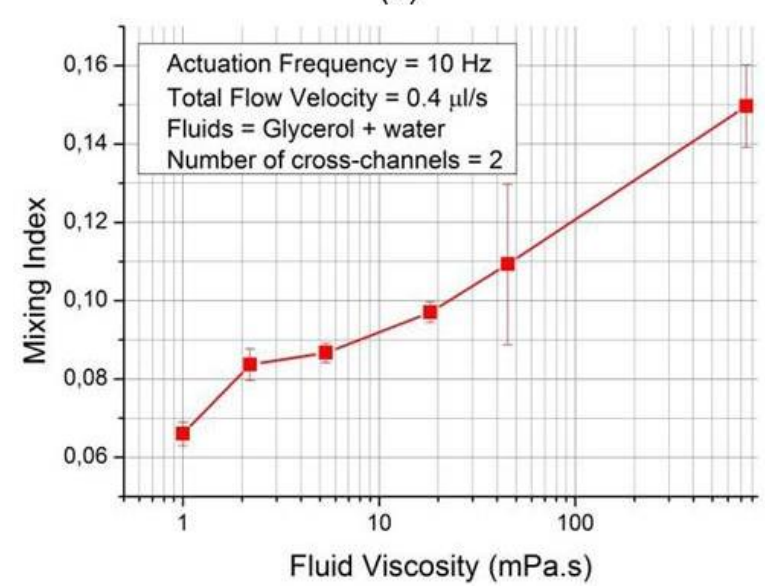

(b)

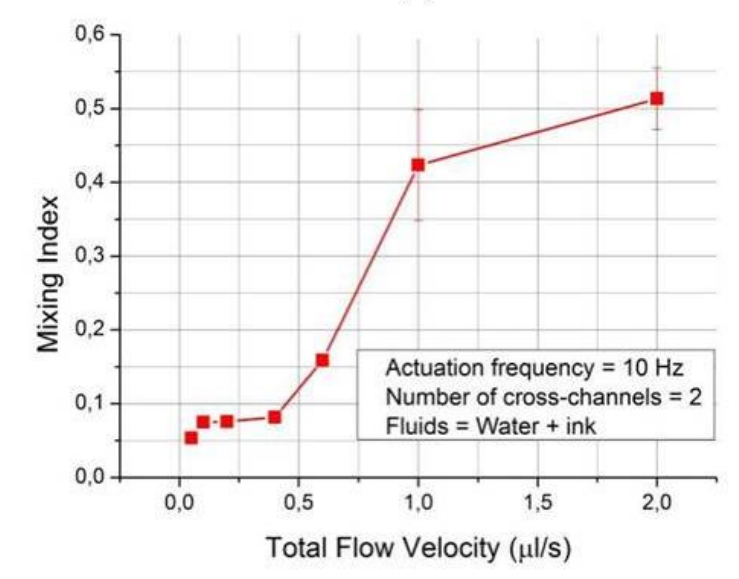

(d)

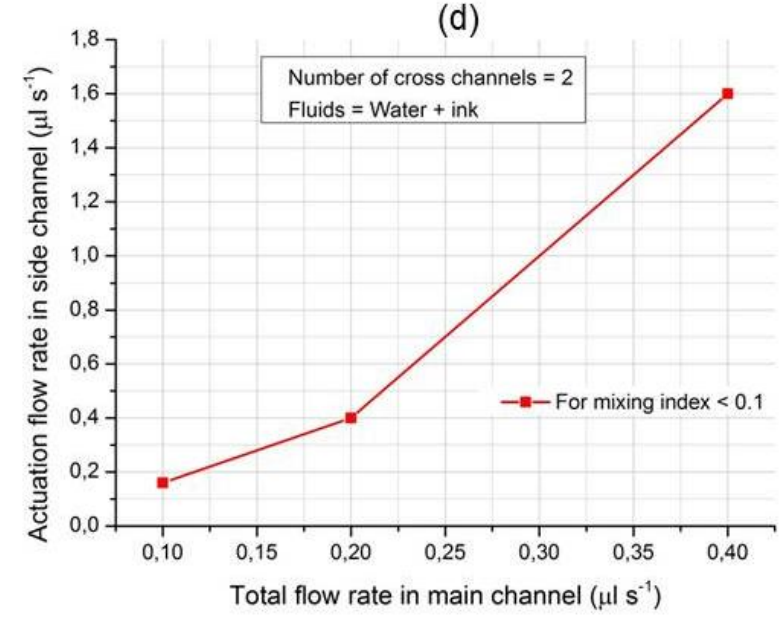

Figure $5 c$ depicts the effect of fluid viscosity on the MI. Fluids with different viscosities were prepared by diluting glycerol at different percentages in DI water. The MI increases (decreasing mixing efficiency) with the increase in the fluid viscosity, which is natural since viscosity is inversely 
proportional to the diffusion constant. Still, for a wide range of viscosities (up to $50 \mathrm{mPa}$ ), the MI is below 0.1 which is the threshold of 'good mixing' commonly used in other studies $[11,18]$.

Transient response of a mixer gives the continuous value of MI during Braille-pin actuation. Such response depicts the mixing speed and steady state MI value of the mixer during actuation. The transient response, of the MI at optimal operating conditions defined through the above-mentioned parameter studies is shown in Figure 6. Here the mixing time is on the order of $10^{2} \mathrm{~ms}(\sim 500 \mathrm{~ms})$, and homogeneous mixing is sustained for as long as the experiment was conducted.

Figure 6. The transient response for MI of the cross channel mixer with optimal design and operational parameters. The MI value reaches 0.1 within $1 \mathrm{~s}$. The steady state error is less than $10 \%$.

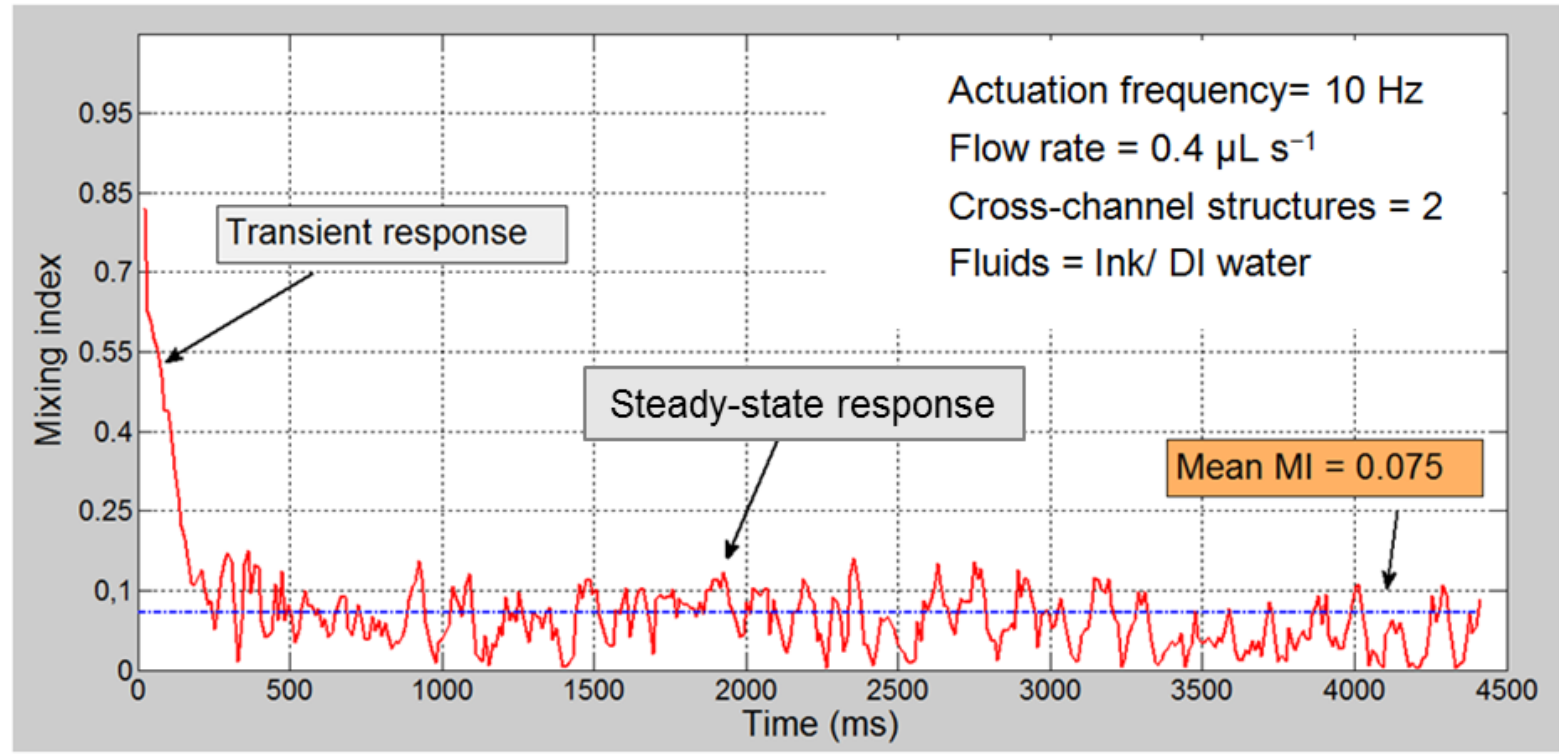

As an application example, dilution of a cell suspension in continuous flow was investigated. The cell suspension was of wild yeast type (Saccharomyces cerevisiae) in YNB medium $\left(10^{6} \mathrm{cells}_{\mathrm{mL}}^{-1}\right.$ ). The photographs of cell dilution near the outlet channel are given in Figure 7. The mixer consists of two cross-channels structures and the actuation frequency is $10 \mathrm{~Hz}$. The total flow rate is $0.4 \mu \mathrm{L} \mathrm{s}{ }^{-1}$, and the flow rate ratio is 1:10 (cell suspension: DI water). The images are taken at the downstream channel of the mixer approximately at $1-\mathrm{mm}$ after the mixing chamber. Initially at $t=0$, which is the instant of no-actuation condition, no mixing is observed and all cells flow in the lower part of the channel in the image. Due to the high viscosity of yeast cell and the medium, the transient response (mixing time) is relatively higher. After $2 \mathrm{~s}$ of actuation the cells are dispersed throughout the width of the channel and diluted. During the mixing operation some yeast cells flow into the side-channels towards pin-actuation area, but no bioaccumulation of the side-channels were observed. Bioaccumulation in side-channels can reduced the mixing efficiency by reducing the actuation pressure. The side-channel flow rate is $c a .1 .6 \mu \mathrm{L} \mathrm{s}^{-1}$ (at $10 \mathrm{~Hz}$ ) which is enough to avoid any bioaccumulation during the mixing operation. After the mixing operation the yeast cells were flushed away with DI water and later immersed in ethanol ultrasonic bath for chip cleaning. The shear stress in the main channel during the induced side-channel flow is roughly calculated to be on the order of $\sim 10^{2} \mathrm{~Pa}$. It is known that a shear-stress of this range does not affect the viability of yeast cells [19]. 
Figure 7. Continuous dilution of yeast cells in the Braille pin actuated micromixer after onset of Braille pin actuation. Snapshots (a), (b) and (c) at the downstream of the first cross-channel 0,1 , and $2 \mathrm{~s}$ after the onset of Braille actuation. Original flow rate ratio of yeast sample and DI water is 1:10 (total flow rate $=0.4 \mu \mathrm{L} \mathrm{s}^{-1}$ ).

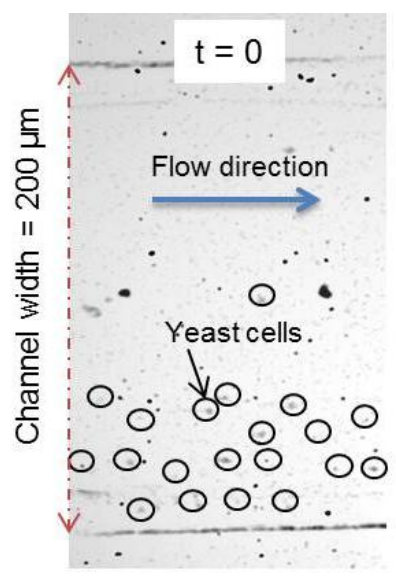

(a)

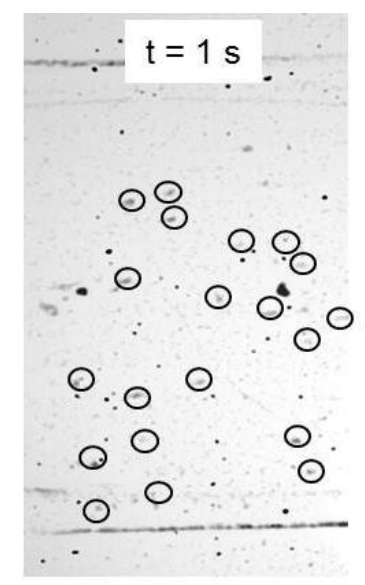

(b)

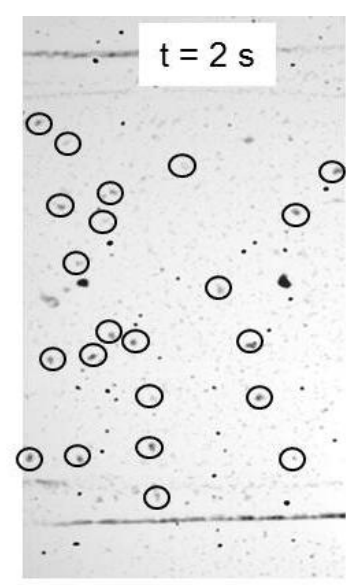

(c)

\section{Conclusions}

We demonstrated a novel microfluidic unit operation for mixing enhancement by membrane actuation using a mechanical Braille pin actuator array. It is shown that rapid mixing of cell/particle flow can be achieved for volume flows in the range up to $2 \mu \mathrm{L} \mathrm{s}^{-1}$ using a cost-effective configuration with easily replaceable PDMS microfluidic chips. Chaotic advection in the main channel is demonstrated by the generation of periodic stretching and folding. For continuously flowing fluids, a MI of 0.1 was achieved by a residence time of half a second using two cross-channels structures in the mixer. Several influencing parameters of the mixing setup have been studied. It is found that parameters like flow rate ratio and fluid viscosity have little to no significant effect on mixing for a wide range, whereas actuation frequency and volume flow has significant effect on mixing efficiency. The successful demonstration of cell sample dilution proved the feasibility of Braille display actuated PDMS micromixers. In future, such micromixers may serve as a microfluidic component in bio-analytical devices or as microfluidic unit operation in Braille pin actuated microfluidic platforms.

\section{Acknowledgments}

The work has received funding from the European Community's Seventh Framework Programme (FP7) as task of the project NANOBE, grant agreement N 227243 (nano and microtechnology based analytical devices for online measurements of bioprocesses). The authors would like to thank the colleagues Taimur Aftab, Henning Meier, and Günter Roth for their help during this work, and Biofluidix $\mathrm{GmbH}$ for providing on-demand Braille interface software and hardware.

\section{References}

1 Nguyen, N.-T. Micromixers, Fundamental, Design and Fabriaction, 2nd ed.; Elsevier: Waltham, MA, USA, 2012. 
2 Jeong, G.S.; Chung, S.; Kim, C.B.; Lee, S.H. Applications of micromixing technology. Analyst 2010, 135, 460-473.

3 Nguyen, N.-T.; Wu, Z. Micromixers-a review. J. Micromech. Microeng. 2005, 15, R1-R16.

4 Tabeling, P.; Chabert, M.; Dodge, A.; Jullien, C.; Okkels, F. Chaotic mixing in cross-channel micromixers. Philos. Transact. A Math. Phys. Eng. Sci. 2004, 362, 987-1000.

5 Gouillart, E.; Dauchot, O.; Thiffeault, J.L. Measures of mixing quality in open flows with chaotic advection. Phys. Fluids 2011, 23, doi:10.1063/1.3506817.

6 Stroock, A.D.; Dertinger, S.K.W.; Ajdari, A.; Mezić, I.; Stone, H.A.; Whitesides, G.M. Chaotic mixer for micro channels. Science 2002, 295, 647-651.

7 Liu, R.H.; Stremler, M.A.; Sharp, K.V.; Olsen, M.G.; Santiago, J.G.; Adrian, R.J.; Aref, H.; Beebe, D.J. Passive mixing in a three-dimensional serpentine microchannel. J. Microelectromech. Syst. 2000, 9, 190-197.

8 Jen, C.P.; Wu, C.Y.; Lin, Y.C. Design and simulation of the micromixer with chaotic advection in twisted micro channels. Lab Chip 2003, 3, 77-81.

9 Xia, H.; Wan, S.; Shu, C.; Chew, Y. Chaotic micromixers using two-layer crossing channels to exhibit fast mixing at low Reynolds numbers. Lab Chip 2005, 5, 748-755.

10 Lu, L.H.; Ryu, K.S.; Liu, C. A magnetic microstirrer and array for microfluidic mixing. $J$. Microelectromech. Syst. 2002, 11, 462-469.

11 Tekin, H.C.; Sivagnanam, V.; Ciftlik, A.T.; Sayah, A.; Vandevyver, C.; Gijs, M.A.M. Chaotic mixing using source-sink microfluidic flows in a PDMS chip. Microfluid. Nanofluid. 2011, 10, 749-759.

12 Lee, Y.K.; Deval, J.; Tabeling, P.; Ho, C.M. Chaotic mixing in electrokinetically and pressure driven micro flows. In Proceedings of the 14th IEEE International Conference on Micro Electro Mechanical Systems, Interlaken, Switzerland, 21-25 January 2001; pp. 483-486.

13 Gu, W.; Zhu, X.; Futai, N.; Cho, B.S.; Takayama, S. Computerized microfluidic cell culture using elastomeric channels and Braille displays. Proc. Natl. Acad. Sci. USA 2004, 101, 15861-15866.

14 Erickson, B.E. Braille pins control microfluidic flow. Anal. Chem. 2005, 77, 93-93.

15 Duffy, D.C.; McDonald, J.C.; Schueller, O.J.A.; Whitesides, G.M. Rapid prototyping of microfluidic systems in poly (dimethylsiloxane). Anal. Chem. 1998, 70, 4974-4984.

16 Futai, N.; Gu, W.; Song, J.W.; Takayama, S. Handheld recirculation system and customized media for microfluidic cell culture. Lab Chip 2005, 6, 149-154.

17 BioFluidix GmbH Web site. Available online: http://www.biofluidix.com (accessed on 3 November 2012).

18 Mao, X.; Juluri, B.K.; Lapsley, M.I.; Stratton, Z.S.; Huang, T.J. Milliseconds microfluidic chaotic bubble mixer. Microfluid. Nanofluid. 2010, 8, 139-144.

19 Lange, H.; Taillandier, P.; Riba, J.P. Effect of high shear stress on microbial viability. J. Chem. Technol. Biotechnol. 2001, 76, 501-505.

(C) 2013 by the authors; licensee MDPI, Basel, Switzerland. This article is an open access article distributed under the terms and conditions of the Creative Commons Attribution license (http://creativecommons.org/licenses/by/3.0/). 